\title{
PERFIL DAS GESTANTES E CONTEÚDO DAS ORIENTAÇÕES SOBRE OS SINAIS DE TRABALHO DE PARTO RECEBIDAS NO PRÉ-NATAL
}

Profile of pregnant and content of the guidelines on sings of labor to give birth

Perfil de mujeres embarazadas y contenido de las directrices sobre señales de trabajo infantil recibidas en prenatal

Lia Cardoso de Aguiar • Enfermeira no Hospital Universitário da Universidade Federal do Maranhão. Doutora em Saúde Coletiva pela Universidade Federal do Maranhão - PGSC/UFMA E-mail: lia_cardoso@hotmail.com

Maria de Lourdes Cardoso Silva • Enfermeira • Especialista em Saúde da Família pela Faculdade Gianna Beretta • E-mail: 1lourdescardoso@hotmail.com

Viviane Santos de Oliveira • Enfermeira • Especialista em Saúde da Família pela Faculdade Gianna Beretta • E-mail: vivianeoliveira27_7@hotmail.com

José Ricardo Rodrigues de Aguiar • Enfermeiro • Especialista em Saúde da Família e em Gestão de Serviços de Saúde • E-mail: aguiaricardo@hotmail.com

Lanna Karla Monroe Dourado • Enfermeira no Hospital São Domingos • Graduada pela Faculdade Santa Terezinha - CEST • E-mail: lanna_monroe@hotmail.com

Autora responsável pela correspondência:

Lia Cardoso de Aguiar lia_cardoso@hotmail.com 


\section{RESUMO}

Introdução: O pré-natal visa preparar para o parto. As orientações recebidas na atenção pré-natal são fundamentais para a vivência saudável durante toda gestação pela gestante e sua família, porém ainda há muitas falhas nestas ações educativas. Objetivo: Conhecer as orientações sobre os sinais de trabalho de parto realizadas para as gestantes durante o pré-natal em uma Unidade Básica de Saúde. Metodologia: Estudo de caráter descritivo com abordagem quantitativa em um Centro de Saúde em São Luís, Maranhão. Para a coleta de dados, utilizou-se um questionário aplicado a uma amostra de 11 gestantes. Resultados: A maioria das gestantes tinha 20 anos ou mais, vivia sem companheiro, com ensino médio completo e renda familiar entre um e dois salários mínimos, tendo como principal ocupação ser do lar. Quanto ao início do pré-natal, 45,4\% no primeiro e 36,4\% no segundo trimestre. Quanto ao número de consultas realizadas, $54,5 \%$ não realizou consulta no primeiro trimestre; $72,8 \%$ realizou pelo menos duas consultas no segundo trimestre; e apenas 18,2\% realizou o mínimo de três consultas preconizado pelo Ministério da Saúde no terceiro trimestre. Neste estudo apenas $27,3 \%$ das gestantes participou de algum grupo de educação em saúde específico para gestantes. E sobre os sinais de trabalho de parto, apenas $45,4 \%$ foi orientada, dentre estas a orientação mais frequente $(60,0 \%)$ continha os principais sinais de trabalho de parto. Conclusões: Este estudo evidencia que as orientações sobre os sinais de trabalho de parto são pouco frequentes e por vezes sequer são fornecidas durante as consultas. Isto pode explicar a escassez de publicações que abordem a temática, embora seja consenso que a educação em saúde no pré-natal é essencial para o fortalecimento da autonomia das gestantes no momento do trabalho de parto e parto.

Palavras-Chave: Trabalho de parto. Gravidez. Cuidado Pré-Natal. Educação em Saúde.

\section{ABSTRACT}

Introduction: Prenatal care is designed to prepare for childbirth. The guidelines received in prenatal care are fundamental for a healthy life throughout pregnancy by the pregnant and her family, but there are still many shortcomings in these as educational actions. Objective: To know the guidelines on the signs of labor to five birth performed for pregnant during prenatal care in a Basic Health Unit. Methodology: Descriptive study with a quantitative approach at a Health Center in São Luís, Maranhão. For the data collection, a questionnaire was applied to a sample of 11 pregnants. Results: The majority of pregnant were 20 years of age or older, living without a partner, with full secondary education and family income between one and two minimum wages, having as their main occupation of the home. As for the onset of prenatal care, $45.4 \%$ were in the first and $36.4 \%$ in the second trimester. Regarding he number of consultations, $54.5 \%$ did not make a referral in the first quarter; $72.8 \%$ erformed at least two visits in the second quarter; and only $18.2 \%$ performed the inimum of three consultations recommended by the Ministry of Health in the third arter. In this study, only $27.3 \%$ of the pregnant women participated in any specific alth education group for pregnant women. And on the signs of labor, $45.4 \%$ received entation, in $60.0 \%$ of these the content of the guidelines was expulsion of the mucus 
plug, loss of amniotic fluid, regular uterine contractions. Conclusions: This study shows that guidelines on signs of labor is uncommon and are sometimes not even made during consultations. This may explain the scarcity of publications that address the theme, although there is a consensus that health education in prenatal care is essential for strengthening the autonomy of pregnant women during labor and delivery.

Keywords: Labor. Pregnancy. Prenatal care. Health education.

\section{RESUMEN}

Introducción: Las pautas recibidas en la atención prenatal son fundamentales para una experiencia saludable durante el embarazo por parte de la mujer embarazada y su familia, sin embargo, todavía hay muchas fallas en estas acciones educativas. Objetivo: Conocer las pautas sobre los signos de parto realizados para mujeres embarazadas durante la atención prenatal en una Unidad Básica de Salud. Metodología: Estudio descriptivo con enfoque cuantitativo en un Centro de Salud en São Luís, Maranhão. Para la recolección de datos, se aplicó un cuestionario a una muestra de 11 mujeres embarazadas. Resultados: La mayoría de las gestantes tenían 20 años o más, vivían sin pareja, con estudios secundarios completos e ingresos familiares entre uno y dos salarios mínimos, siendo la ocupación principal el hogar. En cuanto al inicio de la atención prenatal, $45,4 \%$ en el primer trimestre y $36,4 \%$ en el segundo trimestre. En cuanto al número de consultas realizadas, el 54,5\% no realizó consultas en el primer trimestre; El 72,8\% realizó al menos dos consultas en el segundo trimestre; y solo el $18.2 \%$ tuvo un mínimo de tres consultas en el tercer trimestre. En este estudio, solo el $27.3 \%$ de las mujeres embarazadas participaron en un grupo de educación de salud específico para mujeres embarazadas. Y sobre los signos de parto, solo se aconsejó el $45.4 \%$, entre ellos la guía más frecuente $(60.0 \%)$ contenía los principales signos de parto. Conclusiones: Este estudio muestra que la orientación sobre los signos del trabajo de parto es poco común y, en ocasiones, ni siquiera se brinda durante las consultas. Esto puede explicar la escasez de publicaciones que aborden el tema, aunque existe consenso en que la educación en salud en la atención prenatal es fundamental para fortalecer la autonomía de la gestante durante el trabajo de parto y el parto.

Palabras clave: Trabajo. El embarazo. Cuidado prenatal. Educación para la salud. 


\section{Introdução}

Durante a gestação as mulheres são tomadas por expectativas e medos que permeiam o trabalho de parto que irão influenciar na experiência da parturiçãoํ․

A assistência pré-natal é um importante componente da atenção à saúde das mulheres no período gravídico-puerperal impactando diretamente nos desfechos perinatais, uma vez que as orientações e os cuidados recebidos nesse período estão entre os fatores determinantes do resultado desta vivência².

O pré-natal constitui-se um momento de conhecimentos mas também uma oportunidade para que os profissionais de saúde realizem ações integrais de promoção à saúde como ações educativas que promovam o conhecimento sobre o processo gestacional $^{3}$. No terceiro trimestre, quando as alterações fisiológicas da gravidez ficam mais evidentes, é o momento oportuno para realizar as orientações quanto aos sinais de trabalho de parto e o fim da gestação.

O momento do parto tem sido descrito como complicado, incerto e doloroso o que torna diálogo sobre sinais e sintomas do início do trabalho de parto no pré-natal imprescindível. Informações sobre os procedimentos que serão realizados no parto minimizam a ansiedade e passam segurança com relação aos procedimentos e profissionais ${ }^{2}$.

As orientações no pré-natal são fundamentais para a vivência saudável durante toda gestação pela gestante e sua família. Porém muitas gestantes não recebem orientações durante o pré-natal ${ }^{4}$. E por vezes, quando orientadas os profissionais de saúde não fornecem informações sobre os sintomas fisiológicos da gestação, modificações e cuidado com o próprio corpo, prevenção de doenças, preparo para o parto e outras 5 .

Rios e Vieira ${ }^{3}$ analisaram consultas de enfermagem realizadas em São Luís MA e concluiu que as ações educativas durante o pré-natal eram falhas, uma vez que mesmo numa gestação sem intercorrências e comparecendo a todas as consultas de é-natal, as mulheres finalizavam a gravidez sem conhecimento para vivenciar o mento do parto.

Ainda observa-se consultas de pré-natal baseadas apenas em avaliações de a, especialmente médicas, abordando informações apenas com relação a 
prescrição das medicações e requerimento de exames sem conversas sobre parto na consulta médica ${ }^{6}$.

Neste contexto, este estudo buscou conhecer as orientações sobre os sinais de trabalho de parto realizadas para as gestantes durante o pré-natal em uma Unidade Básica de Saúde (UBS).

\section{Metodologia}

Trata-se de um estudo de caráter descritivo e abordagem quantitativa com o objetivo de conhecer as orientações sobre os sinais de trabalho de parto que são realizadas às gestantes durante o pré-natal. Realizado no período de agosto a outubro de 2015 conforme a demanda de atendimento de pré-natal realizados pelos profissionais médicos e enfermeiros na UBS.

O estudo foi realizado no Centro de Saúde Salomão Fiquene, situado no distrito Cohab, no município de São Luís - MA. Esta unidade de saúde possui uma equipe Estratégia de Saúde da Família que atua na atenção ao pré-natal.

A amostra em estudo foi de conveniência, pela impossibilidade de se obter uma amostra aleatória representativa de mulheres grávidas, pois não há um registro único e confiável dessas mulheres e/ou daquelas que fazem pré-natal. A seleção das gestantes se deu no momento de comparecimento à UBS para realizar a consulta/acompanhamento pré-natal. Nesta ocasião, os pesquisadores realizavam o convite para participar da pesquisa em seguida apresentavam o Termo de Consentimento Livre e Esclarecido para confirmação de participação na pesquisa. Dessa forma, a amostra constituiu-se com 11 gestantes.

Os critérios de inclusão foram ser primíparas com idade gestacional a partir de $33^{\text {a }}$ semanas, com dados suficientes em seus prontuários e cartão da gestante. Não foram inclusas gestantes cuja DUM era desconhecida ou incerta.

Os dados foram coletados por meio de questionário semiestruturado ompreendendo as seguintes variáveis: sociodemográficas (idade, estado civil, colaridade, ocupação e renda familiar); história clínica e obstétrica das gestantes ício do pré-natal, número de consultas por trimestre, participação em grupo de 
gestantes); conhecimento sobre os sinais preditivos do parto (expulsão do tampão mucoso, perda de líquido amniótico e contrações uterinas regulares) ${ }^{7}$. Foi realizada análise descritiva por médias e porcentagens.

A pesquisa foi aprovada pelo Comitê de Ética e Pesquisa da Universidade UNICEUMA em consonância com a Resolução 466/ 2012 do Conselho Nacional de Saúde.

\section{Resultados e Discussão}

$\mathrm{Na}$ amostra de 11 gestantes, $81,8 \%$ tinha 20 anos ou mais, ou seja, adultas e em fase inicial e intermediária de idade reprodutiva. A idade mínima das gestantes estudadas foi de 18 anos e a máxima de 29 anos (Tabela 1).

A faixa etária das gestantes estudadas delineiam-se como uma população jovem, sendo este um aspecto positivo pois sabe-se que existe um impacto da idade da mulher sobre os resultados perinatais: as mulheres chamadas adultas jovens, entre 20 e 35 anos, em geral, tem resultados mais favoráveis 8 , 9 .

No estudo de Souza et al. ${ }^{10}$ também houve um predomínio da faixa etária de 20 a 25 anos (42\%). No entanto, há de se considerar que estudos recentes apontam para frequência maior de gravidez nos extremos de idade. Atualmente as mulheres estão tendo cada vez mais tardiamente filhos. Experenciar a gravidez mais tardiamente tem sido uma tendência crescente entre as mulheres nas últimas décadas devido à sua maior inserção no mercado de trabalho e maior tempo de formação profissional ${ }^{11}$.

A maioria das gestantes vivia sem companheiro (54,5\%), tinha ensino médio completo $(45,4 \%)$, com renda familiar entre 1 e 2 salários mínimos $(81,8 \%)$ e quanto à ccupação, $36,6 \%$ eram do lar. 
Tabela 1 Características sociodemográficas das gestantes assistidas no Centro de Saúde Salomão Fiquene. São Luís - MA, 2015.

\begin{tabular}{|c|c|c|}
\hline VARIÁVEIS & f & $\%$ \\
\hline \multicolumn{3}{|l|}{ Faixa etária } \\
\hline$<20$ anos & 02 & 18,2 \\
\hline 20 anos e + & 09 & 81,8 \\
\hline \multicolumn{3}{|l|}{ Estado Civil } \\
\hline Com companheiro & 05 & 45,5 \\
\hline Sem companheiro & 06 & 54,5 \\
\hline \multicolumn{3}{|l|}{ Escolaridade } \\
\hline Ensino Fundamental completo & 01 & 9,1 \\
\hline Ensino Médio incompleto & 00 & 0,0 \\
\hline Ensino Médio completo & 05 & 45,4 \\
\hline Ensino Superior incompleto & 03 & 27,3 \\
\hline Ensino Superior completo & 02 & 18,2 \\
\hline \multicolumn{3}{|l|}{ Ocupação } \\
\hline Do lar & 04 & 36,3 \\
\hline Professora & 01 & 9,1 \\
\hline Estudante & 01 & 9,1 \\
\hline Secretaria & 01 & 9,1 \\
\hline Antropóloga & 01 & 9,1 \\
\hline Atendente & 03 & 27,3 \\
\hline \multicolumn{3}{|l|}{ Renda Familiar } \\
\hline De 1 até 2 salários mínimos & 09 & 81,8 \\
\hline$>2$ e $<5$ salários mínimos & 01 & 9,1 \\
\hline > 5 salários mínimos & 01 & 9,1 \\
\hline
\end{tabular}

No que diz respeito ao estado civil, estudo realizado em Bonito-PE sobre a percepção das gestantes sobre as consultas médicas e de enfermagem no pré-natal de baixo risco apresentam a maioria das gestantes vivendo com seus companheiros, casadas ou em união estável12. Salienta-se que, a mulher viver sem companheiro durante a gravidez pode complicar o estado emocional trazendo um déficit na strutura econômica familiar, fato que pode trazer risco à gestação, acarretando complicações para o feto ao nascer, dentre eles o baixo peso 9 .

Um estudo ${ }^{13}$ realizado em Fortaleza - CE encontrou uma frequência maior gestantes que viviam com companheiro. Aqui o companheiro da mulher foi siderado o acompanhante ideal no processo de parturição, porém a presença dele parto ainda é pouco frequente. 
No que diz respeito a escolaridade, houve o predomínio de gestantes com ensino médio completo $(45,4 \%)$, seguido por aquelas com ensino superior incompleto $(27,3 \%)$ e superior completo $(18,2 \%)$ demonstrando que a amostra em estudo é escolarizada. Estudo realizado com gestantes atendidas em uma Unidade Básica de Saúde de São Luís - MA no ano de 2013 verificou o nível de escolaridade semelhante ao encontrado neste estudo, ou seja, com predomínio do ensino médio completo (40\%). Contudo, os achados sobre a escolaridade das gestantes na cidade de São Luís estão em dissonância com outros estudos onde a prevalência de gestantes escolarizadas foi baixa $^{14}$.

Em relação à ocupação profissional e à renda familiar das gestantes pesquisadas, verificou-se que $36,3 \%$ eram do lar e $81,8 \%$ possuem renda de 1 a 2 salários mínimos. A situação de ser "do lar" e não ter dupla jornada de trabalho favorece mulheres reduz o risco de apresentar pré-eclâmpsia ${ }^{15}$. Por outro lado, a baixa renda está associada a alguns riscos para a gestação, surgindo indagações sobre instabilidades sociais que podem atuar em situações de riscos à saúde ${ }^{16}$.

Com relação ao início do pré-natal, 45,4\% gestantes relataram início no primeiro trimestre de gestação. Estima-se que um quarto dos óbitos infantis e todos os óbitos maternos decorram da ausência da oferta de pré-natal adequado. Por essa razão, a oferta desse tipo de serviço de forma qualificada é de fundamental importância17.

Quanto ao número de consultas realizadas no $1^{\circ}$ trimestre de gestação $54,5 \%$ não realizaram consulta pré-natal (Tabela 2). O início do pré-natal no primeiro trimestre é excepcionalmente relevante à saúde materna e perinatal, pois facilita detectar uma possível gestação de risco contribuindo para a assistência adequada com desfecho mais propício e positivo, evitando assim complicações no parto ${ }^{18}$.

No $2^{\circ}$ trimestre gestacional, $72,8 \%$ realizou pelo menos duas consultas. Já no $3^{\circ}$ trimestre, apenas $18,2 \%$ realizou o mínimo preconizado pelo Ministério da Saúde (MS). Ainda segundo MS, o pré-natal deve ser iniciado precocemente e regularmente, m no mínimo de seis consultas visando ações adequadas em todo o ciclo gravídico ${ }^{18}$.

As orientações de gestantes em grupo sobre os sinais de trabalho de parto importantes para que as mesmas possam compartilhar informações entre si, tirar idas, relatar seus medos e vivenciarem experiências prévias. As vantagens das 
atividades em grupo são o compartilhamento de experiências e liberdade para expor medos e anseios. Porém, algumas gestantes podem preferir tirar suas dúvidas, desmitificar seus mitos e expor suas vivências de forma particular.

Tabela 2: Caracterização do acompanhamento pré-natal das gestantes assistidas no Centro de Saúde Salomão Fiquene, segundo início do pré-natal, quantidade de consultas, participação em algum grupo de educação em saúde. São Luís - MA, 2015.

\begin{tabular}{lllr}
\hline & VARIÁVEIS & $\mathbf{f}$ & \% \\
\hline Ínicio do pré-natal & & \\
$1^{\mathrm{o}}$ Trimestre & 05 & 45,4 \\
$2^{\mathrm{o}}$ Trimestre & 04 & 36,4 \\
$3^{\mathrm{o}}$ Trimestre & 02 & 18,2
\end{tabular}

\section{Quantidade de consultas}

\section{$1^{0}$ Trimestre}

01

$02 \quad 18,2$

02

$01 \quad 9,1$

03

$02 \quad 18,2$

Nenhuma

$06 \quad 54,5$

\section{$2^{\circ}$ Trimestre}

01

$03 \quad 27,2$

02

$04 \quad 36,4$

03

$3^{\circ}$ Trimestre

$04 \quad 36,4$

01

$05 \quad 45,4$

02

$04 \quad 36,4$

03

$01 \quad 9,1$

04

01

Participação em algum grupo de educação em saúde

Sim

Não

$08 \quad 72,7$

Neste estudo apenas 27,3\% das gestantes participaram de algum grupo de educação em saúde específico para gestantes (Tabela 3).

As ações educativas com grupos de gestantes é uma estratégia que permite permear o universo das grávidas ${ }^{19}$. Vale considerar que as gestantes que não articipam de grupos acabam perdendo a oportunidade de receberem informações ecessárias que, porventura no pré-natal, não puderam ser repassadas. 
Tabela 3: Orientações sobre os sinais de trabalho de parto para as gestantes assistidas no Centro de Saúde Salomão Fiquene. São Luís - MA, 2015.

\begin{tabular}{lcc}
\hline \multicolumn{1}{c}{ VARIÁVEIS } & f & \% \\
\hline Recebeu orientações sobre os sinais de trabalho de parto & & \\
Sim & 05 & 45,4 \\
Não & 06 & 54,6 \\
& & \\
Conteúdo das orientações sobre sinais de trabalho de parto & & \\
$\begin{array}{l}\text { Expulsão do tampão mucoso, perda da bolsa d'água, contrações } \\
\text { uterinas regulares }\end{array}$ & 03 & 60,0 \\
$\begin{array}{l}\text { Perda da bolsa d'água } \\
\text { Contrações uterinas regulares }\end{array}$ & 01 & 20,0 \\
\hline
\end{tabular}

Os grupos de gestantes são fundamentais para suprir os anseios e necessidades das gestantes, contribuindo para troca de experiências, bem como complementando as consultas de pré-natal20. A realização de grupos de apoio à gestante, com a participação de uma equipe de saúde multiprofissional, é fundamental para garantir uma abordagem integral da gestante e sua família, visando troca de saberes, informações sobre o processo de gestação, técnicas de relaxamento, dentre outros.

Sobre as orientações recebidas sobre os sinais de trabalho de parto durante o pré-natal apenas $45,4 \%$ das gestantes estudadas referiram ter sido orientada pelo médico ou enfermeiro. Estudos apontam que a maioria das gestantes entrevistadas relatou não ter recebido orientações sobre os sinais de alerta e de trabalho de parto durante a assistência pré-natal ${ }^{4,21}$. Contrapondo os dados apresentados, há estudos 22 , ${ }^{23}$ que apontam frequências maiores de gestantes que receberam orientações sobre os sinais de trabalho de parto por profissionais da saúde

A orientação da gestante sobre os sinais de trabalho de parto evitará aflições desnecessárias. O MS preconiza que durante o pré-natal a gestante receba orientações em especial relacionadas ao processo gestacional, mudanças corporais e emocionais durante a gravidez, assim também como sinais de trabalho de parto, parto puerpério ${ }^{18}$.

Estudo sobre a contribuição do pré-natal para o parto normal, reafirma o poderamento da mulher quando orientada durante o pré-natal, já a falta de rmação pode gerar preocupações desnecessárias e expectativas frustradas ${ }^{24}$. 
Ainda na tabela 3 observa-se que a orientação mais frequente continha os principais sinais de trabalho de parto. Em Belém-PA, a prévia orientação das grávidas sobre os sinais de trabalho de parto levou a uma proporção bem maior de parto vaginal do que cesarianas e ainda na chegada à maternidade em franco trabalho de parto pela maioria das gestantes ${ }^{25}$.

\section{Conclusões}

O estudo evidenciou que as orientações sobre os sinais de trabalho de parto são pouco frequentes e por vezes sequer são fornecidas durante as consultas. Isto pode explicar a escassez de publicações que abordem a temática, embora seja consenso que a educação em saúde no pré-natal é essencial para o fortalecimento da autonomia das gestantes no momento do trabalho de parto e do parto propriamente dito. Por fim, ratifica-se a necessidade de priorizar as ações educativas durante o pré-natal com especial atenção para as orientações sobre sinais de trabalho de parto.

\section{Referências}

1. Firmino KC, Lima EP, Correia TRL, Silva JCB, Albuquerque NLA. Percepção da mulher frente à dor do Parto. Rev Ciên. Plur. 2020;6(1):87-101.

2. Francisquini AR, Higarashi IH, Serafim D, Bercini LO. Orientações recebidas durante a gestação, parto e pós-parto por um grupo de puérperas. Ciência, Cuid e Saúde. 2010;9(4):743-51.

3. Rios CTF, Vieira NFC. Ações educativas no pré-natal: reflexão sobre a consulta de enfermagem como um espaço para educação em saúde. Cien Saude Colet. 2007;12:477-86.

Félix HCR, Corrêa CC, Matias TGC, Parreira BDM, Paschoini MC, Ruiz MT. Sinais de alerta e de trabalho de parto: conhecimento entre gestantes. Rev Bras Saúde Matern Infant. 2019;19(2):335-41.

Caldeira AP, Oliveira RM, Rodrigues OA. Qualidade da assistência maternoinfantil em diferentes modelos de Atenção Primária. Cien Saude Colet. 2010;15:3139-47. 
6. Souza AQ, Marchiori MRCT, Cabral FB, Diaz CM, Santos NO, Pizolotto ALZ. A assistência no pré-natal no contexto da estratégia de saúde da família sob o olhar do enfermeiro. Rev Eletrônica Acervo Saúde. 2019;(27):e733.

7. Montenegro CAB, Rezende-Filho J. Mecanismo do parto. In: Obstetrícia. 11th ed. Rio de Janeiro: Guanabara Koogan; 2010.

8. Frets RC. Effect of advanced age on fertility and pregnancy in women. 2011.

9. Lima GSP, Sampaio HAC. Influência de fatores obstétricos, socioeconômicos e nutricionais da gestante sobre o peso do recém-nascido: estudo realizado em uma maternidade em Teresina, Piauí. Rev Bras Sade Matern Infant Matern Infant. 2004;4(3):253-61.

10. Souza NA, Queiroz LLC, Queiroz RCCS, Ribeiro TSF, Fonseca MSS. Perfil epidemiológico das gestantes atendidas na consulta de pré-natal de uma Unidade Básica de Saúde em São Luís-MA. Rev Ciências da Saúde. 2013;15(1).

11. Nicésio MG, Oliveira ISB, Alves MI, Alves AC, Carvalho VV, Lenza NFB. Perfil de gestantes atendidas na atenção primária em uma cidade do interior de Minas Gerais. Rev Iniciação Científica da Lib. 2018;8(1):70.

12. Andrade FM, Castro JFL, Silva AV. Percepção das gestantes sobre as consultas médicas e de enfermagem no pré-natal de baixo risco. Rev Enferm do Cent Oeste Min. 2016;6(3):292-300.

13. Holanda SM, Castro RCMB, Aquin PS, Pinheiro AKB, Lopes LG, Martins ES. Influência da participação da companhia no pré - natal: satisfação de primiparas no apoio no nascimento. Texto Context. 2018;27(2).

14. Silva MCRG, Silva LSR, Sousa JO, Frota MCQA, Carneiro JKR, Oliveira MAS. Perfil Epidemiológico-Obstétrico E Sociodemográfico-De Gestantes Atendidas Em Um Centro De Saúde Da Família. Rev Saúde e Desenvolv. 2019;13(14):10011.

15. Moura ERF, Santos OCG, Damasceno AKC, Pereira MQ Fatores de risco para síndrome hipertensiva específica da gestação entre mulheres hospitalizadas com pré-eclâmpsia. Cogitare Enferm. 2010;15(2).

Xavier RB, Jannotti CB, Silva KS, Martins AC. Risco reprodutivo e renda familiar: análise do perfil de gestantes. Cien Saude Colet. 2013;18:1161-71.

Saavedra JS, Cesar JA. Uso de diferentes critérios para avaliação da inadequação do pré-natal: um estudo de base populacional no extremo Sul do Brasil. Cad Saude Publica. 2015;31:1003-14.

Brasil. Atenção ao pré-natal de baixo risco. Cadernos de Atenção Básica. Brasília 
(DF): Ministério da Saúde; 2012.

19. Duarte SJH, Borges AP, Arruda GL. Ações de enfermagem na educação em saúde no pré-natal: relato de experiência de um projeto de extensão da Universidade Federal do Mato Grosso. Rev Enferm do Centro-Oeste Min. 2011;1(2):277-282.

20. Lima ER, Henriques AHB, Batista SSS, Pereira JCN, Barbosa LU, Muniz MLC. Atuação e apoio de familiares no ciclo gravídico-puerperal: vivência prática em um grupo de gestantes. Rev Saúde-UNG-Ser. 2018;11(1 ESP):54.

21. Gonçalves MF, Teixeira ÉMB, Silva MAS, Corsi NM, Ferrari RAP, Pelloso SM, et al. Pré-natal: preparo para o parto na atenção primária à saúde no sul do Brasil. Rev Gaúcha Enferm. 2017;38(3) e63.

22. Lima EC, Santos LM, Santos SC, Christoffel MM, Kerber NPC. Vivências de familiares durante o trabalho de parto pré-termo. Rev Cuid. 2019;10(1).

23. Oliveira LFM, Davim RMB, Alves ÉSRC, Rodrigues ESRC, Nóbrega MF, Torquato JA. Vivência de puérperas adolescentes quanto à gravidez e trabalho de parto. Rev enferm UFPE line. 2016;395-406.

24. Ribeiro JF, Luz VL, Sousa AS, Silva GLL, Feitosa VC, Almeida MF. Contribuição do pré-natal para o parto normal na concepção do enfermeiro da estratégia saúde da família. Rev Interdiscip. 2016;9(1):161-70.

25. Moura AML. A relevância do preparo no pré-natal de primigestas para o trabalho de parto: relato de experiência sobre as consultas puerperais na unidade básica de saúde de Icoaraci. Florianópolis. 2016. 